\title{
Contribution of beverages to daily added sugar intake: compliance with guidelines' recommendations
}

\author{
๑DHande Mortaş, @Saniye Bilici \\ Gazi University, Faculty of Health Sciences, Department of Nutrition and Dietetics, Ankara, Turkey
}

Cite this article as: Mortaş H, Bilici S. Contribution of beverages to daily added sugar intake: compliance with guidelines' recommendations. J Health Sci Med 2021; 4(5): 598-603.

\begin{abstract}
Aim: Beverages that contribute significantly to the daily added sugar intakes are reported to cause increased health problems such as obesity as long as their consumption is not limited. The aim of this study is to estimate added sugar intake from beverages.

Material and Method: This cross-sectional descriptive study conducted on 837 adults aged between 18-65 years. A questionnaire including questions about general socio-demographic characteristics (gender, occupation, income status, age, education) was applied. The beverage consumption within previous month was questioned using a beverage frequency questionnaire. The weight and height of the individuals were measured.

Results: In the present study, the mean added sugar intake from beverages was $13.1 \pm 17.6 \mathrm{~g} / \mathrm{day}$. Fruit drinks and caloric sodas had the highest contributions to daily added sugar intake, followed by sweetened tea and coffee. It was found that $11.95 \%$ and 9.68\% of participants exceeded the recommendation of the American Heart Association and World Health Organization, respectively.

Conclusion: Sugar sweetened beverages are significant contributors to daily added sugar intake in Turkish population. Therefore, decreasing the consumption of sugar sweetened beverages should be main public health implementation to avoid the negative health outcomes including obesity, diabetes, cardiovascular diseases that may occur in the future.
\end{abstract}

Keywords: Added sugar, free sugar, sugar sweetened beverages, fruit drinks, caloric soda

\section{INTRODUCTION}

In recent years, the researchers focus on the intake of free sugars, especially in the form of sugar-sweetened beverages, that increase overall energy intake, may reduce the intake of more nutritionally foods and leading to an unhealthy diet, weight gain and increased risk of non-communicable diseases (1-3). Reduced intake of free sugars throughout the life course is recommended to provide health benefits (4). World Health Organization (WHO) recommends reducing the intake of free sugar below $10 \%$ of total energy. Additionally, the recommendation that reducing the intake of free sugars below $5 \%$ of total energy intake would provide additional health benefits was determined as conditional (5). WHO uses the term of "free sugar" while developing the recommendation, whereas the American Heart Association (AHA) uses the term of "added sugar". The AHA recommends added sugar no more than 25 grams for females and 37 grams for males (6). The terms of free sugar and added sugar are actually quite similar to each other. The difference in the term of free sugar is the inclusion of sugar, which is naturally found in fruit juices and fruit juice concentrates $(5,7)$. Both terms are frequently used in studies assessing the compliance of sugar consumption amounts with the recommendation (8-10). In one of these studies, the median free sugar and added sugar intake are $14 \%$ ( $74 \mathrm{~g} /$ day) and $12 \%$ (64 g/day) of total energy, respectively. Non-alcoholic beverages especially soft drinks are the main sources of free and added sugars in adults (8). Similarly, beverages contribute most (47\%) to added sugar in American diets according to NHANES data (11). Added sugar intake is about $16.3 \%$ of total energy in United States (12). In Canada, the mean added sugar intake is $11-13 \%$ of total energy. The main contributor of added sugar intake is soft drinks 
(13). Therefore, it is mentioned that the consumption of sugar sweetened beverages as "probable contributor" to the increased prevalence of obesity is worth to investigate given the high contribution of soft drinks and the amounts of discretionary beverages including sugar sweetened beverages in diet $(14,15)$. It is important to note that The Heart and Stroke Foundation of Canada declares the free sugar intake should be less than $10 \%$ of total energy by assessing the sugar sweetened beverage consumption patterns obtained from prospective cohort studies in accordance with WHO (16).

In this context, this study aims (1) to estimate added sugar intake from beverages in adults; (2) to describe beverage types which are the main sources of added sugar; (3) to investigate the association of added sugars with demographic descriptors; and (4) to determine the adherence to the recommendations for added sugar.

\section{MATERIAL AND METHOD}

\section{Study Design, Participants and Recruitment}

This cross-sectional descriptive study conducted between June 2019 and October 2019 on 837 adults aged between 18-65 years. The study was carried out in randomly selected individuals living in Ankara, the capital city of Turkey. The individuals who had chronic disease, pregnant and lactating ones were excluded. Consents of the participants were obtained through the voluntary consent form in accordance with the Helsinki declaration. This study was approved by Gazi University Ethics Committee (Date: 14.11.2017, Decision No: 2017439). All procedures were carried out in accordance with the ethical rules and the principles of the Declaration of Helsinki.

A questionnaire including questions about general socio-demographic characteristics (gender, occupation, income status, age, education), the frequency of beverage consumption were applied via face to face interview. The weight (kg; with calibrated device) and height $(\mathrm{cm}$; with portable stadiometer) of the individuals were measured by the researchers to nearest $0.5 \mathrm{~kg}$ and 0.1 $\mathrm{cm}$, respectively. Body mass index (BMI) was calculated as the weight $(\mathrm{kg})$ divided by the squared of height $\left(\mathrm{m}^{2}\right)$. The BMI values were classified into three categories: underweight $\left(<18.5 \mathrm{~kg} / \mathrm{m}^{2}\right)$, normal or healthy weight $\left(18.5-24.9 \mathrm{~kg} / \mathrm{m}^{2}\right)$, and overweight or obese $(\geq 25.0 \mathrm{~kg} /$ $\left.\mathrm{m}^{2}\right)(17)$.

\section{Instruments, Measures, and Procedures}

The beverage consumption within previous month was questioned using a beverage frequency questionnaire which was applied in similar researches (18-20) and adapted to Turkish by the researchers. Frequency categories were responded on never, daily, weekly or monthly basis and then the amount or serving size of each beverage reported was asked. Name brands of the beverages consumed were also provided to determine the added sugar intake more reliably. Frequency ("How often") is converted to the unit of times per day, then multiplied by the amount consumed ("How much each time") to provide average daily beverage consumption in $\mathrm{mL}$ per day.

Added sugars in the mean amount of beverages consumed per day were calculated by modeling the methods of Louie et al. (21) 2015. The adapted method to calculate added sugar in the beverages is presented in the Figure 1. Gray box indicates decision end points. When information of the added sugar content was limited similar to Step 5 in Figure 1, the added sugar was calculated as sugar content of the beverage minus natural occurring sugar estimated by comparison with unsweetened variety by modeling the methods of Sluik et al. (8) 2016 . In this step, different method was used from Louie et al. (21) 2015. However, similar results were obtained. In the present study, the steps up to Step 5 in Figure 1 were sufficient for the added sugar calculation. There was no need to use Step 6 in Figure 1.

Free sugars were calculated by collecting added sugars with the sugars naturally present in fruits juices and fruit concentrates. The prevalence of excessive added sugar intake from beverages was assessed using the AHA's recommendation (25 grams for females and 37 grams for males). Total energy requirement was used to calculate how much percentage of the daily energy met from free sugar in the beverages according to the free sugar intake recommendation of WHO (below 10\% of total energy). The equations proposed by Schofield in 1985 were selected according to the age and gender of the participants to calculate basal metabolic rate (22); then multiplied it by the corresponding physical activity level depending on their occupation to estimate the individuals' energy requirements (23).

\section{Data Analysis}

The data were evaluated using SPSS 22.0 statistic package program software. Mean and standard deviation were obtained using descriptive statistics. The data providing parametric condition was evaluated using independent sample $t$ test to describe the mean differences and significance. Chi-square test was used to predict the differences between categorical parameters. The findings were determined within a $95 \%$ confidence interval and in the $\mathrm{p}<0.01$ and $\mathrm{p}<0.05$ significance level. The participants met the recommendations was presented using percentages. The compliance two different recommendations for added sugar intake in making the distinction by evaluating the participants as excess sugar intake and recommended level of sugar intake was assessed using the kappa test. 


\begin{tabular}{|c|c|}
\hline \multicolumn{2}{|c|}{ Step $1.0 \mathrm{~g}$ total sugar is in the beverage. } \\
\hline No=go to step 2 & Yes $=$ Added sugar $=0$ \\
\hline \multicolumn{2}{|c|}{$\downarrow$} \\
\hline \multicolumn{2}{|c|}{$\begin{array}{l}\text { Step 2. The beverage is defined as one of the no added sugars } \\
\text { beverage. }\end{array}$} \\
\hline No=go to step 4 & Yes=go to step 3 \\
\hline \multicolumn{2}{|c|}{$\downarrow$} \\
\hline \multicolumn{2}{|c|}{$\begin{array}{l}\text { Step 3. A food suitable for the definition of } 100 \% \text { added sugar is } \\
\text { putted into the beverage before consumption. }\end{array}$} \\
\hline $\begin{array}{l}\text { Yes }=\text { Added sugar }=100 \% \text { of the } \\
\text { food putted into the beverage. }\end{array}$ & No $=$ Added sugar $=0$ \\
\hline \multicolumn{2}{|c|}{$\downarrow$} \\
\hline \multicolumn{2}{|c|}{$\begin{array}{l}\text { Step 4. The added sugar content of the beverage is known } \\
\text { (handmade or taken from the nutrition label). }\end{array}$} \\
\hline $\begin{array}{l}\text { Yes=Added sugar=calculate the } \\
\text { added sugar based on the label } \\
\text { or recipe. }\end{array}$ & No $=$ go to step 5 \\
\hline & \\
\hline \multicolumn{2}{|c|}{ Step 5. There is a comparable unsweetened variety of the beverage. } \\
\hline No=go to step 6 & $\begin{array}{c}\text { Yes }=\text { Calculate added sugar by } \\
\text { comparison with unsweetened } \\
\text { variety. }\end{array}$ \\
\hline & \\
\hline \multicolumn{2}{|c|}{ Step 6. There is analytical data of sugar types available. } \\
\hline $\begin{array}{l}\text { No=Subjectively estimate the } \\
\text { added sugar content. } \\
\text { (using similar products, } \\
\text { available information or } 50 \% \text { of } \\
\text { the total sugars as added sugar) }\end{array}$ & $\begin{array}{l}\text { Yes }=\text { Estimate based on } \\
\text { analytical data }\end{array}$ \\
\hline
\end{tabular}

Figure 1. Flow diagram adapted from Louie et al., 2015 for assessment added sugar in beverages (21).

\section{RESULTS}

\section{Demographic Descriptors, Sugar Sweetened Beverages Consumption and Added Sugar Intake}

The demographic descriptors, sugar sweetened beverage consumption and the mean added sugar intake from beverages of the participants were presented in Table 1 . The mean amounts of sugar sweetened beverage (SSB) consumption and added sugar intake from beverages was $232.5 \pm 327.7 \mathrm{~mL} /$ day and $13.1 \pm 17.6 \mathrm{~g} / \mathrm{d}$, respectively. In general, males had higher the daily consumption of $\mathrm{SSB}$, added sugar intake from beverages than females ( $\mathrm{p}$ $<0.001)$. According to age groups, the amounts of SSB consumption and added sugar intake from beverages were higher in older participants (18-30 years) than the other age group (31-65 years) $(p<0.001 ; p<0.05$, respectively). Although in low socioeconomic level, both the amounts of SSB consumption $(267.8 \pm 311.7 \mathrm{~mL} /$ day $)$ and added sugar intake from beverages $(15.4 \pm 22.1 \mathrm{~g} /$ day) were higher than the other socioeconomic levels, the difference was not statistically significant $(\mathrm{p}>0.05)$. Individuals with higher education level consumed more SSB than in that of lower education level even though not statistically significant. Added sugar intake from beverages of the individuals at higher education level was more, significantly $(\mathrm{p}<0.001)$. According to BMI, overweight and obese were found to consume more SSB and added sugar $(\mathrm{p}>0.05)$. The differences among the BMI categories were not significant ( $p>0.05)$.

\begin{tabular}{|c|c|c|c|}
\hline Characteristics & n (\%) & 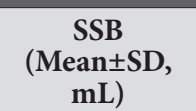 & $\begin{array}{r}\text { Added Sugar } \\
(\text { Mean } \pm \text { SD, g) }\end{array}$ \\
\hline Total participants & $837(100)$ & $232.5 \pm 327.7$ & $13.1 \pm 17.6$ \\
\hline \multicolumn{4}{|l|}{ Gender } \\
\hline Males & $191(22.8)$ & $340.8 \pm 449.6^{* *}$ & $18.9 \pm 25.4^{* *}$ \\
\hline Females & $646(77.2)$ & $200.5 \pm 274.1$ & $11.3 \pm 14.0$ \\
\hline \multicolumn{4}{|l|}{ Age (Years) } \\
\hline $18-30$ & $757(90.4)$ & $221.9 \pm 305.2$ & $12.5 \pm 15.1$ \\
\hline $31-65$ & $80(9.6)$ & $332.4 \pm 483.8^{\star *}$ & $18.1 \pm 32.5^{*}$ \\
\hline \multicolumn{4}{|l|}{ Socioeconomic level } \\
\hline Low & $220(26.3)$ & $267.8 \pm 311.7$ & $15.4 \pm 22.1$ \\
\hline Medium & $541(64.6)$ & $214.9 \pm 323.6$ & $12.1 \pm 15.7$ \\
\hline High & $76(9.1)$ & $255.9 \pm 390.7$ & $13.5 \pm 15.1$ \\
\hline \multicolumn{4}{|l|}{ Education } \\
\hline$\leq 8$ years & $50(6.0)$ & $189.9 \pm 320.1$ & $8.1 \pm 12.6$ \\
\hline$>8$ years & $787(94.0)$ & $235.2 \pm 328.2$ & $13.3 \pm 17.8^{* *}$ \\
\hline \multicolumn{4}{|l|}{ BMI $\left(\mathrm{kg} / \mathrm{m}^{2}\right)$} \\
\hline Underweight & $99(11.8)$ & $216.5 \pm 267.4$ & $13.3 \pm 16.5$ \\
\hline Normal weight & $551(65.8)$ & $234.9 \pm 335.9$ & $12.8 \pm 15.6$ \\
\hline Overweight and obese & $187(22.4)$ & $234.0 \pm 333.3$ & $13.6 \pm 22.9$ \\
\hline
\end{tabular}

\section{Daily Energy and Added Sugar Intake from Beverages}

Mean daily energy and added sugar intake from beverages according to all participants and to only consumers were presented in Table 2. Plain milk was preferred to sweetened milk beverages by $96.3 \%$ of the participants. The mean percentage of the participants consumed $100 \%$ fruit juice was $34.4 \%$ while $43.8 \%$ preferred to consume fruit drinks. The mean percentages of individuals who consumed sweetened coffee and tea, and caloric soda was found $49.5 \%$ and $56.0 \%$, respectively.

The beverages with the highest consumption amounts were unsweetened tea and coffee $(281.4 \pm 379.8 \mathrm{~mL} /$ day), plain milk $(160.6 \pm 193.4 \mathrm{~mL} /$ day $)$ and sweetened tea and coffee $(153.9 \pm 285.6 \mathrm{~mL} /$ day $)$ according to mean consumption of all participants, respectively. On the other hand, unsweetened tea and coffee $(430.6 \pm 395.6$ $\mathrm{mL} /$ day), sweetened tea and coffee $(311.1 \pm 340.7 \mathrm{~mL} /$ day), plain milk ( $166.7 \pm 194.4 \mathrm{~mL} /$ day $)$ and low caloric soda $(160.5 \pm 327.1 \mathrm{~mL} /$ day $)$ were the beverages with highest consumption according to only consumers. Plain milk contributed $\sim 79.5 \mathrm{kcal}$ to the diet. Caloric soda was the beverage providing the second highest level energy; $25.4 \mathrm{kcal}$ came from caloric soda which had the 
most contribution compared to other sugar sweetened beverages according to all participants. Additionally, when examining the mean energy intake in only consumers, all of the sugar sweetened beverages were determined to contribute to energy with similar averages excluding beverages contain naturally occurred sugar such as plain milk and sweetened milk beverages.

Contribution of added sugar to total energy requirement was $\sim 1 \%$ in consumers. Caloric soda contributed the most energy to total energy requirement with the percentage of $1.8 \%$.

In Figure 2, the assessment was based on the added sugar and free sugar received from the beverages only. Although the assessment only included the sugar from beverages, $11.95 \%$ and $9.68 \%$ of participants exceeded the recommendation of AHA and WHO, respectively.

The majority of the participants $(88.05 \%$ and $90.32 \%)$ were found not to exceed the recommendation of AHA and WHO, respectively (Figure 2). According to the kappa statistical results, the agreement between the recommendations of $\mathrm{WHO}$ and AHA was significant ( $\mathrm{p}<0.001$, Figure 2 ), and the agreement rate was $87.0 \%$ between them.

\section{DISCUSSION}

In the present study, the mean added sugar intake from beverages was $13.1 \pm 17.6 \mathrm{~g} /$ day. Males, individuals aged between 31-65 years and at the higher education level had the highest added sugar intake from beverages. Fruit drinks and caloric sodas had the highest contributions from added sugars to total energy requirements, followed by sweetened tea and coffee.
Thompson et al., 2009 were found males to have higher added sugar intake than females according to the NHANES 2003-2004 data, similar to the results of present study. In another study, the added sugar intake of males was higher than females (10), similarly. On the other hand, the youngest age group had higher added sugar intake (10), unlike the present study.

The mean amounts of added sugar intakes were 52 $\mathrm{g}$ in Canadian adults (13), $77 \mathrm{~g}$ in US adults (23), $64 \mathrm{~g}$ in Dutch adults per day (8). Direct comparisons of the present study with other studies are difficult not only because of the assessment of added sugar intake from only beverages in this study, but also because of the variability in definitions. Added sugar definition was used more frequently in the studies $(1,7,13,14,24-26)$ while in others, free sugar definition was evaluated (810).

- Individuals exceed the recommendation - Individuals do not exceed the recommendation

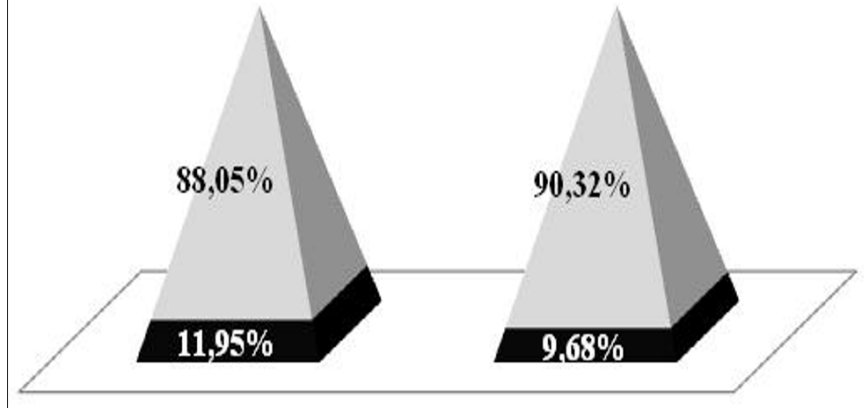

AHA recommendation WHO recommendation

Figure 2. Comparison of added sugar and free sugar intakes of individuals with AHA and WHO recommendations.

Table 2. Daily energy and added sugar intake from beverages

\begin{tabular}{|c|c|c|c|c|c|c|c|c|c|}
\hline \multirow[t]{2}{*}{ Beverages } & \multirow[t]{2}{*}{$\begin{array}{c}\text { Consumers } \\
\mathbf{n}(\%)\end{array}$} & \multicolumn{2}{|c|}{$\begin{array}{l}\text { Amount of beverages } \\
(\text { Mean } \pm \text { SD }, \mathrm{mL} / \mathrm{d})\end{array}$} & \multicolumn{2}{|c|}{$\begin{array}{c}\text { Energy intake } \\
(\text { Mean } \pm \text { SD, kcal/d) }\end{array}$} & \multicolumn{2}{|c|}{$\begin{array}{l}\text { Added sugar intake } \\
\quad(\text { Mean } \pm S D, g / d)\end{array}$} & \multicolumn{2}{|c|}{$\begin{array}{l}\text { Contribution of added } \\
\text { sugar to total energy } \\
\text { requirement } \\
(\text { Mean } \pm S D, \%)\end{array}$} \\
\hline & & Total & Consumers & Total & Consumers & Total & Consumers & Total & Consumers \\
\hline Water & $836(99.8)$ & $1518.1 \pm 670.2$ & $1519.9 \pm 668.6$ & - & - & - & - & - & - \\
\hline \multicolumn{10}{|l|}{ Milk } \\
\hline Sweetened milk beverages & $44(5.3)$ & $1.9 \pm 35.0$ & $37.5 \pm 150.2$ & $4.1 \pm 73.7$ & $78.7 \pm 315.5$ & $0.3 \pm 2.1$ & $5.1 \pm 7.9$ & $0.04 \pm 0.3$ & $0.7 \pm 0.9$ \\
\hline Plain milk & $806(96.3)$ & $160.6 \pm 193.4$ & $166.7 \pm 194.4$ & $79.5 \pm 90.5$ & $82.6 \pm 90.1$ & - & - & - & - \\
\hline \multicolumn{10}{|l|}{ Fruit beverages } \\
\hline$\% 100$ fruit juices & $288(34.4)$ & $22.0 \pm 75.8$ & $63.9 \pm 118.6$ & $10.1 \pm 34.9$ & $29.4 \pm 54.6$ & - & - & - & - \\
\hline Fruit drinks & $367(43.8)$ & $32.7 \pm 85.2$ & $74.5 \pm 116.1$ & $16.3 \pm 42.6$ & $37.2 \pm 58.0$ & $3.6 \pm 9.4$ & $8.2 \pm 12.8$ & $0.6 \pm 1.5$ & $1.5 \pm 2.1$ \\
\hline \multicolumn{10}{|l|}{ Tea and coffee } \\
\hline Sweetened tea and coffee & $414(49.5)$ & $153.9 \pm 285.6$ & $311.1 \pm 340.7$ & $15.4 \pm 28.5$ & $31.1 \pm 34.1$ & $3.8 \pm 7.1$ & $7.8 \pm 8.5$ & $0.7 \pm 1.1$ & $1.3 \pm 1.4$ \\
\hline Unsweetened tea and coffee & $547(65.4)$ & $281.4 \pm 379.8$ & $430.6 \pm 395.6$ & $2.7 \pm 4.2$ & $4.1 \pm 4.7$ & - & - & - & - \\
\hline \multicolumn{10}{|l|}{ Soda } \\
\hline Caloric & $469(56.0)$ & $42.4 \pm 110.6$ & $75.6 \pm 139.1$ & $25.4 \pm 66.4$ & $45.4 \pm 83.4$ & $4.4 \pm 11.4$ & $7.8 \pm 14.3$ & $1.1 \pm 2.3$ & $1.8 \pm 2.9$ \\
\hline Low calorie & $28(3.3)$ & $5.4 \pm 65.5$ & $160.5 \pm 327.1$ & $1.0 \pm 13.0$ & $32.1 \pm 65.4$ & - & - & - & - \\
\hline Alcoholic beverages & $129(15.4)$ & $13.7 \pm 73.0$ & $88.9 \pm 167.5$ & $6.8 \pm 35.8$ & $45.1 \pm 82.2$ & - & - & - & - \\
\hline Sports and energy drinks & $59(7.0)$ & $1.8 \pm 10.4$ & $25.4 \pm 30.6$ & $0.7 \pm 4.1$ & $10.1 \pm 12.2$ & $0.2 \pm 1.2$ & $2.9 \pm 3.6$ & $0.03 \pm 0.2$ & $0.4 \pm 0.5$ \\
\hline
\end{tabular}


The mean intake of added sugars did not exceed the recommendations. However, consumers who exceeded the recommendation of AHA and WHO constituted onetenth of all participants despite considering the added sugars the only from beverages. This rate is thought to be only the tip of the iceberg; such a finding was obtained although amounts of added sugar were assessed from only beverages. In New Zealand, 58\% of adults exceeded this recommendation (10). In study conducted by Sluik et al. (8), 2016, $29-33 \%$ of the adults adhered to the WHO recommendation. In another study in 4140 Australian children and adolescents, $18 \%$ of the participants were consistent with the WHO recommendation (25). Studies examined the compliance of added sugar intake with the recommendation is very limited. In the present study, according to added sugar intake from beverages, 11.95\% and $9.68 \%$ of participants exceeded the recommendation of AHA and WHO, respectively. In Turkey, the study evaluating added and free sugar intakes has not been conducted yet according to our present knowledge. If dietary intakes of individuals are assessed, this ratio is likely to be higher. On the other hand, many studies evaluated the effects of added sugar on health with evidences of sugar sweetened beverages consumption (27-29). Moreover, the authorities including The Heart and Stroke Foundation of Canada and United States Department of Agriculture $(16,17)$ developed recommendations for added sugar intake based on studies assessing the sugar sweetened beverage consumption of populations (27-30). Similarly, in studies assessed the added sugar intake has found that sugar sweetened beverages were the main contributors to added sugar intake $(8,14)$. Therefore, the percentages and amounts of added sugar intake obtained from this study is thought to reflect the consumption of the population. Additionally, SSB is determined as a suitable indicator to develop public health policy because SSB is one of the causes of obesity and empty calorie source $(15,31)$. This health effect was shown in many prospective cohort studies, weight gain and obesity had association with SSB consumption (32-34). However, in this study, there was no difference in the amounts of added sugar intake between BMI groups.

In this study, the main contributor of added sugar intake was caloric soda, followed by fruits drinks and sugar sweetened tea and coffee. The consumption of sugar-free tea or coffee instead of sugar sweetened beverages has positive health effects. In cohort studies, tea and coffee was determined to have associations with lower risk of cardiovascular disease and type 2 diabetes $(34,35)$. Additionally, $100 \%$ fruit juices are healthy alternatives to fruit drinks provided that 4-6 oz ( 118.3-177.4 mL) does not exceed (31). The consumption of diet soda instead of caloric soda affect health more positively $(36,37)$, but it is also stated that it should be approached cautiously (31).

\section{Limitations}

First one of the limitations of this study was the beverages consumption was assessed according to a non-validated questionnaire instead of $24 \mathrm{~h}$ dietary recall. Secondly, the added sugar intake from overall diet was not calculated because of the previous limitation. Thirdly, as in all added sugar studies, obtaining the added sugar contain of some beverages was difficult. Despite the study limitations, it is worth to mention that this is the first study to assess the added sugar intakes among Turkish adults.

\section{CONCLUSION}

The results of this study support the evidence that sugar sweetened beverages are significant contributors to added sugar intake. Added sugar intake from beverages was higher than the recommended levels in $10 \%$ of the participants. Studies should be conducted to provide further support to limit consumption of these beverages in place of healthy alternatives such as water to reduce obesity-related chronic disease risk in Turkish population.

\section{ETHICAL DECLARATIONS}

Ethics Committee Approval: This study was approved by Gazi University Ethics Committee (Date: 14.11.2017, Decision No: 2017439).

Informed Consent: Because the study was designed retrospectively, no written informed consent form was obtained from patients.

Referee Evaluation Process: Externally peer-reviewed.

Conflict of Interest Statement: The author(s) declared no potential conflicts of interest with respect to the research, authorship, and/or publication of this article.

Financial Disclosure: The authors have not declared a specific grant for this research from any funding agency in the public, commercial or not-for-profit sectors.

Author Contributions: All of the authors declare that they have all participated in the design, execution, and analysis of the paper, and that they have approved the final version.

\section{REFERENCES}

1. Lula EC, Ribeiro CC, Hugo FN, Alves CM, Silva AA. Added sugars and periodontal disease in young adults: an analysis of NHANES III data. Am J Clin Nutr 2014; 100: 1182-7.

2. Te Morenga LA, Howatson AJ, Jones RM, Mann J. Dietary sugars and cardiometabolic risk: systematic review and meta-analyses of randomized controlled trials of the effects on blood pressure and lipids. Am J Clin Nutr 2014; 100: 65-79.

3. Singh GM, Micha R, Khatibzadeh S, et al. Estimated Global Burden of Diseases Nutrition and Chronic Diseases Expert Group (NutriCoDE). Estimated global, regional, and national disease burdens related to sugar-sweetened beverage consumption in 2010. Circulation 2015; 132: 639-66. 
4. World Health Organization. Information note about intake of sugars recommended in the WHO guideline for adults and children. Geneva, Switzerland: WHO Document Production Services; 2015a.

5. https://www.who.int/nutrition/publications/guidelines/sugar_ intake_information_note_en.pdf Accessed May 15, 2021.

6. World Health Organization. Guideline: Sugars intake for adults and children. ISBN: 9789241549028. Geneva, Switzerland: WHO Document Production Services; 2015b. Accessed April 30, 2021.

7. The American Heart Association. Dietary sugars intake and cardiovascular health a scientific statement from the American Heart Association. Circulation 2009; 120: 1011-20.

8. Erickson J, Slavin J. Are restrictive guidelines for added sugars science based? Nutrition 2015; 14: 124.

9. Sluik D, van Lee L, Engelen AI, Feskens EJ. Total, free, and added sugar consumption and adherence to guidelines: the Dutch National Food Consumption Survey 2007-2010. Nutrients 2016 8: 70 .

10. Mok A, Ahmad R, Rangan A, Louie JCY. Intake of free sugars and micronutrient dilution in Australian adults. Am J Clin Nutr 2018 107: 94-104.

11. Kibblewhite R, Nettleton A, McLean R, et al. Estimating free and added sugar intakes in New Zealand. Nutrients 2017; 9: 1292.

12. United States Department of Agriculture (USDA). Scientific Report of the 2015 Dietary Guidelines Advisory Committee. https://health.gov/dietaryguidelines/2015-scientific-report/ pdfs/scientific-report-of-the-2015-dietary-guidelines-advisorycommittee.pdf Accessed May 10, 2021.

13. Newens KJ, Walton J. A review of sugar consumption from nationally representative dietary surveys across the world. J Hum Nutr Diet 2016; 29: 225-40.

14. Brisbois TD, Marsden SL, Anderson GH, Sievenpiper JL. Estimated intakes and sources of total and added sugars in the Canadian diet. Nutrients 2014; 6: 1899-912.

15. Sanchez-Pimienta TG, Batis C, Lutter CK, Rivera JA. Sugarsweetened beverages are the main sources of added sugar intake in the Mexican population. J Nutr 2016; 146: 1888-96.

16. World Health Organization/Food and Agriculture Organization. Diet, nutrition and the prevention of chronic diseases-report of a Joint WHO/FAO Expert Consultation. Geneva: World Health Organization; 2003.

17. http://apps.who.int/iris/bitstream/handle/10665/42665/ WHO TRS_916.pdf?sequence=1 Accessed April 30, 2021.

18. The Heart and Stroke Foundation of Canada (HSFC). Position Statement : Sugar, heart disease and stroke; 2014. Accessed April 25, 2021.

19. WHO expert consultation. Appropriate body-mass index for Asian populations and its implications for policy and intervention strategies. Lancet 2004; 363: 157-63.

20. West DS, Bursac Z, Quimby D, et al. Self-reported sugarsweetened beverage intake among college students. Obesity 2006; 14: 1825-31.

21. Berkey CS, Rockett HRH, Field AE, Gillman MW, Colditz GA. Sugar-added beverages and adolescent weight change. Obes Res 2004; 12: 778-88.

22.Opoku-Acheampong AA, Kidd T, Adhikari K, Muturi N, Kattelmann K. Assessing physical activity, fruit, vegetable, and sugar-sweetened beverage intake patterns of college students in Kansas. J Nutr Educ Behav 2018; 50: 977-83.

23. Louie JCY, Moshtaghian H, Boylan S, et al. A systematic methodology to estimate added sugar content of foods. Eur J Clin Nutr 2015; 69: 154-61.

24. Schofield WN. Predicting basal metabolic rate, new standards and review of previous work. Hum Nutr Clin Nutr 1985; 39C: 5-41.
25.WHO. Energy and protein requirements: Report of a joint FAO/ WHO/UNU expert consultation. WHO Technical Report Series No. 724. Geneva; 1985. http://www.fao.org/3/a-y5686e.pdf Accessed October 18, 2020.

26. Ervin RB, Ogden CL. Consumption of added sugar among U.S. adults, 2005-2010. NCHS Data Brief 2013; 122: 1-8.

27.Louie JCY, Moshtaghian H, Rangan AM, Flood VM, Gill TP. Intake and sources of added sugars among Australian children and adolescents. Eur J Nutr 2016; 55: 2347-55.

28. Thompson FE, McNeel TS, Dowling EC, et al. Interrelationships of added sugars intake, socioeconomic status, and race/ ethnicity in adults in the United States: National Health Interview Survey, 2005. J Am Diet Assoc 2009; 109: 1376-83.

29. Singh GM, Micha R, Katibzadeh S, et al. Mortality due to sugar sweetened beverage consumption: A global, regional and national comparative risk assessment. Circulation 2018; 127: AMP22.

30.Buhler S, Raine KD, Arango M, Pellerin S, Neary NE. Building a strategy for obesity prevention one piece at a time: The case of sugar-sweetened beverage taxation. Can J Diabete 2013; 37: $97-$ 102.

31. Malik VS, Popkin BM, Bray GA, Despres JP, Hu FB. Sugar sweetened beverages, obesity, type 2 diabetes mellitus and cardiovascular disease risk. Circulation 2010; 121: 1356-64.

32. Kristal RB, Blank AE, Wylie-Rosett J, Selwyn PA. Factors associated with daily consumption of sugar-sweetened beverages among adult patients at four federally qualified health centers, Bronx, New York, 2013. Prev Chronic Dis 2015; 12: E02.

33.Hu FB. Resolved: there is sufficient scientific evidence that decreasing sugar-sweetened beverage consumption will reduce the prevalence of obesity and obesity-related diseases. Obes Rev 2013; 14: 606-19.

34.Lim L, Banwell C, Bain C, et al. Sugar sweetened beverages and weight gain over 4 years in a Thai national cohort-a prospective analysis. PLoS One 2014; 9: e95309.

35. Mozaffarian D, Hao T, Rimm EB, Willett WC, Hu FB. Changes in diet and lifestyle and long-term weight gain in women and men. N Engl J Med 2011; 364: 2392-2404.

36. O'Connor L, Imamura F, Lentjes MA, et al. Prospective associations and population impact of sweet beverage intake and type 2 diabetes, and effects of substitutions with alternative beverages. Diabetologia 2015; 58: 1474-83.

37. Bhupathiraju SN, Pan A, Manson JE, et al. Changes in coffee intake and subsequent risk of type 2 diabetes: three large cohorts of US men and women. Diabetologia 2014; 57: 1346-54.

38. Ma J, Jacques PF, Meigs JB, et al. Sugar-sweetened beverage but not diet soda consumption is positively associated with progression of insulin resistance and prediabetes. J Nutr 2016; 146: 2544-50.

39. Carwile JL, Willett WC, Spiegelman D, et al. Sugar-sweetened beverage consumption and age at menarche in a prospective study of US girls. Hum Reprod 2015; 30: 675-83. 